\title{
The transfer of alternative tasks in reciprocal cooperation
}

\author{
Manon K. Schweinfurth ${ }^{1,2}$ and Michael Taborsky ${ }^{1}$ \\ ${ }^{1}$ Behavioural Ecology, Institute of Ecology and Evolution, University of Bern, Wohlenstrasse 50a, CH-3032 Hinterkappelen, Switzerland \\ ${ }^{2}$ Correspondence: manon.schweinfurth@iee.unibe.ch / phone: +41 316319151
}

Direct reciprocity can establish stable cooperation. Nevertheless, the significance of this mechanism is yet unclear. A frequent assumption is that both commodity and context should be the same when help is exchanged between social partners. Yet, an exchange of different favours appears more likely in a natural setting. This is assumed to be cognitively demanding, however, because experienced help in one context needs to change the motivation to help by different means or in a different context. We tested whether Norway rats, Rattus norvegicus, transfer help from one cooperative task to another. Individuals could provide food to previously either cooperating or defecting partners by using a different mechanism to produce food for their partner than the partner had used to help them. Test subjects indeed helped previously cooperative partners more often than defecting ones by using a different provisioning mechanism. This implies that rats realize the cooperative propensity of social partners, which they consequently reward by help of a different kind; hence, they do not merely copy experienced helping behaviour. Our results suggest that animals other than primates are capable of transferring help between different contexts, which highlights new possibilities for the occurrence of reciprocal altruism involving different commodities and services in nature.

Keywords: food sharing, gratitude, iterated prisoner's dilemma, Norway rats, reciprocity, trading 


\section{Introduction}

The evolution and maintenance of cooperative interactions between unrelated individuals can be explained by the reciprocal exchange of roles between participants (Taborsky, Frommen, \& Riehl, 2016; Trivers, 1971). Individuals showing direct reciprocity help those that have previously helped them (Axelrod \& Hamilton, 1981). Many experimental studies have demonstrated direct reciprocity in nonhuman animals (e.g. Amici et al., 2014; Carter \& Wilkinson, 2013; Rutte \& Taborsky, 2008, for a review see Taborsky et al., 2016) but its importance in nature has been questioned (Clutton-Brock, 2009; Hammerstein, 2003). Usually, the concept of reciprocity is applied only to situations where the same social service or commodity is returned to the same social partner in a similar context. However, there is no reason to assume that the occurrence of reciprocity is confined to such narrowly defined conditions. More likely, help or social service received in one context should increase the propensity to help a partner also in a different context in the future. This may apply when social partners have changed, such as in generalized reciprocity (Barta, McNamara, Huszár, \& Taborsky, 2011; Pfeiffer, Rutte, Killingback, Taborsky, \& Bonhoeffer, 2005; Rutte \& Taborsky, 2008), or when the opportunity to return a received favour to the same partner has changed, for instance if a different task is required. Experimental studies involving given and received favours in different tasks are rare, despite growing correlative evidence (Carter \& Wilkinson, 2013; Fraser \& Bugnyar, 2012; Romero \& Aureli, 2008).

Preconditions for direct reciprocity include individual recognition and memory of the outcome of past interactions with specific social partners, which is thought to be cognitively demanding (Brosnan, Salwiczek, \& Bshary, 2010; Stevens \& Hauser, 2004). In addition, cooperating across different services implies (1) an understanding of help received from a specific individual and (2) transferring this information to a different service in order to pay back the other, previously received service. There is good evidence that some nonhuman animals exchange different cooperative services reciprocally (reviewed in Taborsky et al., 2016). In monkeys, for example, grooming often seems to be traded against other social services (Borgeaud \& Bshary, 2015; Cheney et al., 2010; Fruteau et al., 2009; 
Hemelrijk, 1994). Experiments on free-ranging vervet monkeys, Chlorocebus pygerythrus, showed that individuals respond more strongly to solicitations for aid of unrelated social partners that had groomed them before (Seyfarth \& Cheney, 1984), and that individuals providing food to the group are subsequently groomed more often by group members (Fruteau et al., 2009).

Although trading different services is very widespread in humans and therefore seems intuitive to us, it may be cognitively demanding (Stevens \& Gilby, 2004). When, for example, grooming is exchanged for social support (Seyfarth \& Cheney, 1984), that is, individual $X$ grooms $Y$ (= service A) and $Y$ will support $X$ in a fight against $Z$ later on (= service $B$ ), this implies both (1) the perception of somehow corresponding payoffs of services $A$ and $B$, and (2) that a cooperative state is associated with a specific individual. If the association of a cooperative state with specific individuals is dynamic, that is, it is updated accordingly over a series of interactions, this would enable group members to cooperate reciprocally with specific partners over different contexts based either on associative learning or on a change in attitudes (de Waal, 2000) or emotions (Schino, di Sorrentino, \& Tiddi, 2007); this works without needing to keep track of the exact outcome of every interaction. In contrast, copying the helpful behaviour of a specific partner would imply memory about, and a specific response to, every single interaction, which would hardly lead to cooperation across different contexts.

Norway rats, Rattus norvegicus, help social partners to obtain food if they have been helped in a similar way by the same or different individuals, that is, they show direct and generalized reciprocity in an iterated prisoner's dilemma situation (Dolivo \& Taborsky, 2015b; Rutte \& Taborsky, 2007, 2008; Schneeberger, Dietz, \& Taborsky, 2012; Wood, Kim, \& Li, 2016). Rats are highly social animals, forming groups of various sizes (McGuire, Pizzuto, Bemis, \& Getz, 2006; Telle, 1966), sharing food (Barnett \& Spencer, 1951) and coordinating cooperative behaviour (Schuster, 2002). There is evidence that they show transitive inference, that is, if an option $A$ is more valuable than $B$ and $B$ is more valuable than C, then A is perceived to be also more valuable than C (Roberts \& Phelps, 1994). Hence, Norway rats seem to be an ideal model to test for the propensity of animals to return received favours by different 
tasks. In this study, we expanded an established experimental paradigm where rats provide food to a social partner by pulling a movable platform by a second, similarly intuitive mechanism, through which food can be delivered to the partner by pushing a lever. Thus, focal rats experienced social partners as cooperating 'pushers' and defecting 'nonpushers', as well as cooperating 'pullers' and defecting 'nonpullers' in a full factorial design. Thereafter, the focal rats always had access to the alternative mechanism to that of their partners to provide food for them. If rats realize the difference between cooperating and defecting providers, we predicted that they should be more likely to use an alternative food-provisioning mechanism to help a previously cooperative partner than a previously defecting partner.

\section{Methods}

\section{Experimental subjects and holding conditions}

We used outbred wild-type adult (1-year-old) female Norway rats (source: Animal Physiology Department, University of Groningen, the Netherlands) weighing on average $300 \mathrm{~g}$. The rats were habituated to handling right after weaning and hence did not show any signs of stress when being handled, transported to the experimental cage and exposed to the set-up. They were individually colour marked and housed with littermates in groups of five sisters in order to provide a social environment (Sharp, Zammit, Azar, \& Lawson, 2003). The cages (80x50 cm and $37.5 \mathrm{~cm}$ high) were separated from each other by opaque walls to exclude visual contact between the groups. In accordance with the animal welfare legislation of Switzerland (Tierschutzverordnung Schweiz 04/2008) we enriched the cages with various materials (a wooden house and board, a tunnel, a piece of wood to nibble, a cardboard roll to play with, digging material (wood shavings), nest-building material (hay) and a salt block, as suggested by established animal care guidelines (Forbes, Blom, Kostmitsopoulus, Moore, \& Perretta, 2007). Food (conventional rat pellets) and water were provided ad libitum. In addition, rats received a corn mix, fresh fruits or vegetables once a day. The ambient 
temperature was $20^{\circ} \mathrm{C} \pm 1{ }^{\circ} \mathrm{C}$, with a relative humidity of $50-60 \%$ and a $12: 12 \mathrm{~h}$ light:dark cycle with lights on at 2000 hours and $30 \mathrm{~min}$ of dawn and dusk. As rats are primarily nocturnal, all training and experiments were conducted during the dark phase under red light (Norton, Culver, \& Mullenix, 1975).

\section{Pre-experimental training}

The experimental set-up was based on a two-player sequential food exchange task (de Waal \& Berger, 2000; Rutte \& Taborsky, 2007). Test cages ( $80 \times 50 \mathrm{~cm}$ and $37.5 \mathrm{~cm}$ high) were divided in half by a wire mesh. All focal and partner rats experienced the following training prior to the experiment.

\section{Pulling task}

First, every rat was trained to pull a stick that was connected to a movable platform in order to receive a food item (one oat flake). The experimenter moved the platform consistently out of the cage over several training sessions, teaching the rats to pull the stick to reach the reward. In the second part of the training, a sister of the rat was placed in the second cage compartment. From then on, the rats (termed donors) did not get a reward themselves for pulling the platform; instead, only their partner (the recipient) received one. The roles of donor and recipient were regularly exchanged, and the intervals between these alternations were increased gradually from switching the roles after each pull to switching them after series of pulls lasting up to $7 \mathrm{~min}$, which corresponds to the experimental period (see Dolivo \& Taborsky, 2015a for a detailed plan of the training scheme). We noted the individuals pulling the most in 12 cages. Afterwards, we randomly chose six of these 12 individuals to serve as 'cooperative partners' in the experiment. In addition, six rats were trained to refrain from pulling by blocking the platform during the $7 \mathrm{~min}$ of exposure to the social partner in the training phase. These six rats were randomly chosen from the original population, and they served as defectors in the experiment.

\section{Pushing task}

This training was also divided into two parts. First, rats were trained to push a lever, which opened a connected trapdoor in a tube, through which a reward was delivered into the cage from above (see 
Appendix Fig. A1). In the second training phase, the rats were again paired with a sister. Similar to the training of the pulling task, from then on the rats never received a reward for themselves when pushing the lever. Again, the same 12 partner rats were trained to be either cooperators or defectors.

\section{Test procedure}

During the experience phase, focal rats experienced cooperators producing rewards by either pulling the platform into the focal rat's compartment or by pushing a lever so that food dropped into the cage from above. Defectors did not produce any food. During the test phase, the roles were exchanged and focal rats could now produce food for their social partner (cooperator or defector) by pushing the lever or pulling the platform. In all cases, rats had to use the alternative mechanism to the one they had experienced before, thus making pure copying of actions impossible. Each focal rat $(N=28)$ was tested four times with two different cooperators and two different defectors, either with the pulling or the pushing task (Fig. 1). Focal rats were unrelated and had never met the social partners (cooperator and defector) prior to the experiment. The treatment order (cooperator or defector and social partner pushes or pulls) was randomly chosen using the Excel command 'RANDBETWEEN'.

\section{Behavioural data}

During both phases, pushes and pulls were counted and the latency to the first event was measured. All focal rats (eight of the original 28 tested rats) that nibbled the wooden stick during the 'pulling' task were excluded from all analyses, as we were unable to exclude potential selfish motivations underlying the operation of the apparatus. A previous study showed that nibbling the stick increases pulling rates while the pulling frequency has no clear relation to the social context (Schweinfurth \& Taborsky, 2016).

\section{Statistical analyses}

To test whether focal rats reciprocate help by different tasks, we analysed the total number of donations using a generalized liner mixed model. We assumed a Poisson distribution as the dependent variable was count data. We included cooperation level (cooperator or defector) and order of 
mechanisms (push $\rightarrow$ pull or pull $\rightarrow$ push) as main factors. Focal identity was included as a random effect because focal rats were tested repeatedly in all four situations. We checked for overdispersion, which was not the case. For the latency to donate the first time we analysed the data using a time to event analysis (Cox proportional hazard model). We included the cooperation level of partners (cooperating versus defecting) and the task (partner pulled versus pushed) as fixed factors. Further, we included the identity of focal rats as a frailty term to account for multiple testing. To simplify the models, we removed the nonsignificant interactions between the main effects (Engqvist, 2005). All statistical analyses were performed using R (version 2.15.2; packages 'Ime4' and 'survival'; The R Foundation for Statistical Computing, Vienna, Austria, http://www.r-project.org).

\section{Ethical note}

The housing of the animals and the experimental procedure were authorized by the Swiss Federal Veterinary Office under licence BE25/14. There was no possibility of physical contact between the test individuals, as a wire mesh separated the experimental compartments, and no injuries occurred.

\section{Results}

Focal rats helped their previously cooperative partners more often than their previously defecting partners (GLMM: $\beta=-0.293 \pm 0.108, z=-2.702, N=20, P=0.007$; Fig. 2 ). Rats did not differ in their response to partners that had either 'pushed' or 'pulled' food for them during the experience phase (GLMM: $\beta=-0.070 \pm 0.107, z=-0.651, N=20, P=0.51$; Fig. 2 ; the performance of all animals during all trials is shown in Appendix Fig. A2).

The latencies to start helping cooperating or defecting partners did not differ (Cox proportional hazard model: coefficient $\left.=-0.051 \pm 0.254, \chi^{2}{ }_{1}=0.04, N=20, P=0.84\right)$. Overall, rats pulled approximately $30 \mathrm{~s}$ earlier than they pushed to help partners (Cox proportional hazard model: coefficient $=1.265 \pm 0.277$, $\chi^{2}{ }_{1}=20.81, N=20, P<0.001$; see Appendix and Appendix Fig. A3). 


\section{Discussion}

Our results demonstrate that Norway rats can generalize their behavioural response to received help. Receiving help raises the cooperation propensity of rats towards the provider irrespective of the task by which it can be returned, which may be based on 'gratitude' (de Waal \& Brosnan, 2006; McCullough, Kimeldorf, \& Cohen, 2016) or an adjustment of the general 'attitude' of an individual towards a social partner (de Waal, 2000). The results cannot be explained as an undirected increase in food delivery propensities after experiencing cooperative partners, as in similar experimental setups rats have been repeatedly shown to supply very little after receiving help from a partner when the neighbouring compartment was empty (Rutte \& Taborsky, 2007, 2008; Schweinfurth \& Taborsky, 2016). To the best of our knowledge, this is the first experimental evidence that animals other than primates tested in a variant of the iterated prisoner's dilemma situation generalize their own cooperation experience by reciprocating received service through a different task.

The cognitive process underlying this accomplishment might appear to be highly challenging. Indeed, reciprocal cooperation has been assumed to be cognitively too demanding to be shown among nonhuman animals (Hammerstein, 2003; Stevens \& Hauser, 2004), especially when involving different currencies (Stevens \& Gilby, 2004). However, complex cognitive capacities are not necessarily involved in such reciprocal exchange. Assigning a trait, attitude or emotion to certain group members based on their previous behaviour can reduce costs of future interactions in many different contexts. For instance, by determining and remembering the fighting ability of previously met opponents, risk of injury can be reduced by avoiding escalated conflicts with certain partners in the future. Similar benefits may accrue from avoiding previously mated conspecifics. Likewise, previous amicable behaviour received from a social partner may change the attitude of a subject towards this individual (attitudinal reciprocity: Brosnan \& de Waal, 2002; de Waal, 2000). Furthermore, receiving help may modulate emotions on a longer timescale (Schino et al., 2007). Both attitudinal and emotionally based reciprocity can account for cooperation shown across contexts, without being cognitively more 
demanding than cooperation exchanged within the same type of service (see also Schino \& Aureli, 2010). Such ability seems very beneficial; therefore the capability of transferring the consequence of social experience between contexts is likely to be under positive selection, as has been suggested for monkeys (Seyfarth \& Cheney, 2003). Norway rats live in variable, often large and complex groups characterized by stable dominance hierarchies (Adams \& Boice, 1983), which affects access to food and mates (Whishaw \& Whishaw, 1996). Therefore, understanding each other's dominance position, for example, seems beneficial to avoid needless conflict and to compete successfully for resources. Similar benefits might ensue from knowing each other's propensity to cooperate, and associating this with different social contexts.

It has been argued that Pavlovian association might suffice to explain the ability of rats to reciprocate received help (Zentall, 2015). In addition, rats are known to commonly copy conspecific behaviour, including the imitation of observed behaviours (Zentall \& Levine, 1972). However, our results showing that rats receiving help by one task return it with a different service clearly rule out that reciprocity in rats merely reflects copying behaviour. Furthermore, even if Pavlovian conditioning might be involved in the behavioural regulation of the rats' response to received help, it cannot account for the observed transfer between different tasks as shown by our results (see Dolivo, Rutte, \& Taborsky, 2016 for additional arguments that mere Pavlovian association processes cannot explain reciprocal cooperation in rats). Further, rats have been experimentally shown to also reciprocate allogrooming, a naturally occurring behaviour where no training is involved; this again suggests that reciprocal cooperation in rats cannot be a mere by-product of conditioning (Schweinfurth, Stieger, \& Taborsky, 2017).

Contingency between different cooperative tasks as observed in our wild-type Norway rats is frequently observed in humans (Jaeggi \& Gurven, 2013; Jaeggi, Hooper, Beheim, Kaplan, \& Gurven, 2016) and might also exist in other animals. Simpler cognitive mechanisms than those used in calculating outcomes, such as attitudinal, symmetry-, or emotion-based mechanisms, may also 
underlie the reciprocal help across different services in other species where correlative evidence suggests such trading (Carter \& Wilkinson, 2013; Fraser \& Bugnyar, 2012; Romero \& Aureli, 2008). The presumption that reciprocal cooperation among nonhuman animals is rare (Clutton-Brock, 2009; Hammerstein, 2003; Stevens, Cushman, \& Hauser, 2005) is probably more apparent than real (Carter, 2014; Taborsky, 2013; Taborsky et al., 2016). It may partly result from the fact that reciprocity is difficult to detect when it takes place between different commodities or social contexts. In this study, we used the same currency, food, to investigate reciprocal trading. By using different currencies in future studies, it can be clarified whether rats also trade different commodities reciprocally, for instance grooming for food.

\section{Conclusion}

Our results show that Norway rats respond to the cooperation levels of social partners and transfer experienced help to a different cooperative task, which suggests behavioural generalization and reciprocal trading. This might reflect a mechanism allowing for appropriate responses to the cumulative information about the social behaviour of different group members in a complex society, setting the stage for group members to negotiate and trade different commodities and services among each other.

\section{Acknowledgments}

We thank Evi Zwygart for help caring for the animals, Markus Wyman for building the test apparatus, Nina Gerber for assistance in training the rats in the first task, Valentina Balzarini for drawings, Karin Schneeberger for comments on the manuscript and Joachim Frommen for helpful discussions. Funding was provided by SNF-grant 31003A_156152 to M.T. 


\section{References}

Adams, N., \& Boice, R. (1983). A longitudinal study of dominance in an outdoor colony of domestic rats. Journal of Comparative Psychology, 97(1), 24-33.

Amici, F., Aureli, F., Mundry, R., Amaro, A. S., Barroso, A. M., Ferretti, J., \& Call, J. (2014). Calculated reciprocity? A comparative test with six primate species. Primates, 55(3), 447-457.

Axelrod, R., \& Hamilton, W. D. (1981). The evolution of cooperation. Science, 211, 1390-1396.

Barnett, S. A., \& Spencer, M. M. (1951). Feeding, social behaviour and interspecific competition in wild rats. Behaviour, 3(3), 229-242.

Barta, Z., McNamara, J. M., Huszár, D. B., \& Taborsky, M. (2011). Cooperation among non-relatives evolves by state-dependent generalized reciprocity. Proceedings of the Royal Society $B$, 278(1707), 843-848.

Borgeaud, C., \& Bshary, R. (2015). Wild vervet monkeys trade tolerance and specific coalitionary support for grooming in experimentally induced conflicts. Current Biology, 25, 3011-3016.

Brosnan, S. F., \& de Waal, F. B. M. (2002). A proximate perspective on reciprocal altruism. Human Nature, 13(1), 129-152.

Brosnan, S. F., Salwiczek, L. H., \& Bshary, R. (2010). The interplay of cognition and cooperation. Philosophical Transactions of the Royal Society B, 365(1553), 2699-2710.

Carter, G. G. (2014). The reciprocity controversy. Animal Behavior and Cognition, 1(3), 368-386.

Carter, G. G., \& Wilkinson, G. S. (2013). Food sharing in vampire bats: reciprocal help predicts donations more than relatedness or harassment. Proceedings of the Royal Society B, 280, 20122573.

Cheney, D. L., Moscovice, L. R., Heesen, M., Mundry, R., \& Seyfarth, R. M. (2010). Contingent cooperation between wild female baboons. Proceedings of the National Academy of Sciences of the United States of America, 107(21), 9562-9566.

Clutton-Brock, T. (2009). Cooperation between non-kin in animal societies. Nature, 462(7269), 51-57.

de Waal, F. B. M. (2000). Attitudinal reciprocity in food sharing among brown capuchin monkeys. Animal Behaviour, 60(2), 253-261.

de Waal, F. B. M., \& Berger, M. L. (2000). Payment for labour in monkeys. Nature, 404, 563.

de Waal, F. B. M., \& Brosnan, S. F. (2006). Simple and complex reciprocity in primates. In P. M. Kappeler \& C. P. van Schaik (Eds.), Cooperation in primates and humans: mechanisms and evolution (Vol. 6, pp. 85-105). Heidelberg, Germany: Springer.

Dolivo, V., Rutte, C., \& Taborsky, M. (2016). Ultimate and proximate mechanisms of reciprocal altruism in rats. Learning and Behavior, 44, 223.

Dolivo, V., \& Taborsky, M. (2015a). Cooperation among Norway rats: the importance of visual cues for reciprocal cooperation, and the role of coercion. Ethology, 121, 1071-1080.

Dolivo, V., \& Taborsky, M. (2015b). Norway rats reciprocate help according to the quality of help they received. Biology Letters, 11, 20140959.

Engqvist, L. (2005). The mistreatment of covariate interaction terms in linear model analyses of behavioural and evolutionary ecology studies. Animal Behaviour, 70(4), 967-971.

Forbes, D., Blom, H., Kostmitsopoulus, N., Moore, G., \& Perretta, G. (2007). Euroguide on the accommodation and care of animals used for experimental and other scientific purposes. London, U.K.: Federation of European Laboratory Animal Science Associations. 
Fraser, O. N., \& Bugnyar, T. (2012). Reciprocity of agonistic support in ravens. Animal Behaviour, 83(1), 171-177.

Fruteau, C., Voelkl, B., van Damme, E., \& Noë, R. (2009). Supply and demand determine the market value of food providers in wild vervet monkeys. Proceedings of the National Academy of Sciences of the United States of America, 106(29), 12007-12012.

Guez, D., \& Audley, C. (2013). Transitive or not: a critical appraisal of transitive inference in animals. Ethology, 119(9), 703-726.

Hammerstein, P. (2003). Why is reciprocity so rare in social animals? In P. Hammerstein (Ed.), Genetic and cultural evolution of cooperation (pp. 83-93). Cambridge, MA: MIT Press.

Hemelrijk, C. K. (1994). Support for being groomed in long-tailed macaques, Macaca fascicularis. Animal Behaviour, 48, 479-481.

Jaeggi, A. V., \& Gurven, M. (2013). Reciprocity explains food sharing in humans and other primates independent of kin selection and tolerated scrounging: a phylogenetic meta-analysis. Proceedings of the Royal Society B, 280(1768), 20131615.

Jaeggi, A. V., Hooper, P. L., Beheim, B. A., Kaplan, H., \& Gurven, M. (2016). Reciprocal exchange patterned by market forces helps explain cooperation in a small-scale society. Current Biology, 26(16), 35-57.

McCullough, M. E., Kimeldorf, M. B., \& Cohen, A. D. (2016). An adaption for altruism? Current Directions in Psychological Science, 17(4), 281-285.

McGuire, B., Pizzuto, T., Bemis, W. E., \& Getz, L. L. (2006). General ecology of a rural population of Norway rats (Rattus norvegicus) based on intensive live trapping. The American Midland Naturalist, 155(1), 221-236.

Norton, S., Culver, B., \& Mullenix, P. (1975). Development of nocturnal behavior in albino rats. Behavioral Biology, 15(3), 317-331.

Pfeiffer, T., Rutte, C., Killingback, T., Taborsky, M., \& Bonhoeffer, S. (2005). Evolution of cooperation by generalized reciprocity. Proceedings of the Royal Society B, 272(1568), 1115-1120.

Roberts, W. A., \& Phelps, M. T. (1994). Transitive inference in rats: a test of the spatial coding hypothesis. Psychological Science, 5(6), 368-374.

Romero, T., \& Aureli, F. (2008). Reciprocity of support in coatis (Nasua nasua). Journal of Comparative Psychology, 122(1), 19-25.

Rutte, C., \& Taborsky, M. (2007). Generalized reciprocity in rats. PLoS Biology, 5(7), 1421-1425.

Rutte, C., \& Taborsky, M. (2008). The influence of social experience on cooperative behaviour of rats (Rattus norvegicus): direct vs generalised reciprocity. Behavioral Ecology and Sociobiology, 62(4), 499-505.

Schino, G., \& Aureli, F. (2010). A few misunderstandings about reciprocal altruism. Communicative and Integrative Biology, 3(6), 561-563.

Schino, G., di Sorrentino, E. P., \& Tiddi, B. (2007). Grooming and coalitions in Japanese macaques (Macaca fuscata): partner choice and the time frame reciprocation. Journal of Comparative Psychology, 121(2), 181-188.

Schneeberger, K., Dietz, M., \& Taborsky, M. (2012). Reciprocal cooperation between unrelated rats depends on cost to donor and benefit to recipient. BMC Evolutionary Biology, 12(1), 41.

Schuster, R. (2002). Cooperative coordination as a social behaviour: experiments with an animal model. Human Nature, 13(1), 47-83.

Schweinfurth, M. K., Stieger, B., \& Taborsky, M. (2017). Experimental evidence for reciprocity in 
allogrooming among wild-type Norway rats. Scientific Reports, 7: 4010.

Schweinfurth, M. K., \& Taborsky, M. (2016). No evidence for audience effects in reciprocal cooperation of Norway rats. Ethology, 122, 513-521.

Seyfarth, R. M., \& Cheney, D. L. (1984). Grooming, alliances and reciprocal altruism in vervet monkeys. Nature, 308, 541-543.

Seyfarth, R. M., \& Cheney, D. L. (2003). The structure of social knowledge in monkeys. In F. B. M. de Waal \& P. L. Tyack (Eds.), Animal Social Complexity (pp. 207-229). Cambridge, MA: Harvard Universtity Press.

Sharp, J., Zammit, T., Azar, T., \& Lawson, D. (2003). Stress-like responses to common procedures in individually and group-housed female rats. Journal of American Association for Laboratory Animal Science, 42(1), 9-18.

Stevens, J. R., Cushman, F. A., \& Hauser, M. D. (2005). Evolving the psychological mechanisms for cooperation. Annual Review of Ecology, Evolution, and Systematics, 36(1), 499-518.

Stevens, J. R., \& Gilby, I. C. (2004). A conceptual framework for nonkin food sharing: timing and currency of benefits. Animal Behaviour, 67(4), 603-614.

Stevens, J. R., \& Hauser, M. D. (2004). Why be nice? Psychological constraints on the evolution of cooperation. Trends in Cognitive Sciences, 8(2), 60-65.

Taborsky, M. (2013). Social evolution: reciprocity there is. Current Biology, 23(11), 486-488.

Taborsky, M., Frommen, J. G., \& Riehl, C. (2016). The evolution of cooperation based on direct fitness benefits. Philosophical Transactions of the Royal Society B, 371, 20150472.

Telle, H. (1966). Beitrag zur Erkenntnis der Verhaltensweise von Ratten, vergleichend dargestellt bei Rattus norvegicus und Rattus rattus. Zeitschrift Für Angewandte Zoologie, 53, 129-196.

Trivers, R. L. (1971). The evolution of reciprocal altruism. The Quarterly Review of Biology, 46(1), 3557.

Whishaw, I. Q., \& Whishaw, G. E. (1996). Conspecific aggression influences food carrying: studies on a wild population of Rattus norvegicus. Aggressive Behavior, 22(1), 47-66.

Wood, R. I., Kim, J. Y., \& Li, G. R. (2016). Cooperation in rats playing the iterated Prisoner's dilemma game. Animal Behaviour, 114, 27-35.

Zentall, T. R. (2015). Reciprocal altruism in rats: why does it occur? Learning and Behavior, 44(7), 1516.

Zentall, T. R., \& Levine, J. M. (1972). Observational learning and social fascilitation in the rat. Science, 178(4066), 1220-1221. 
Experience phase
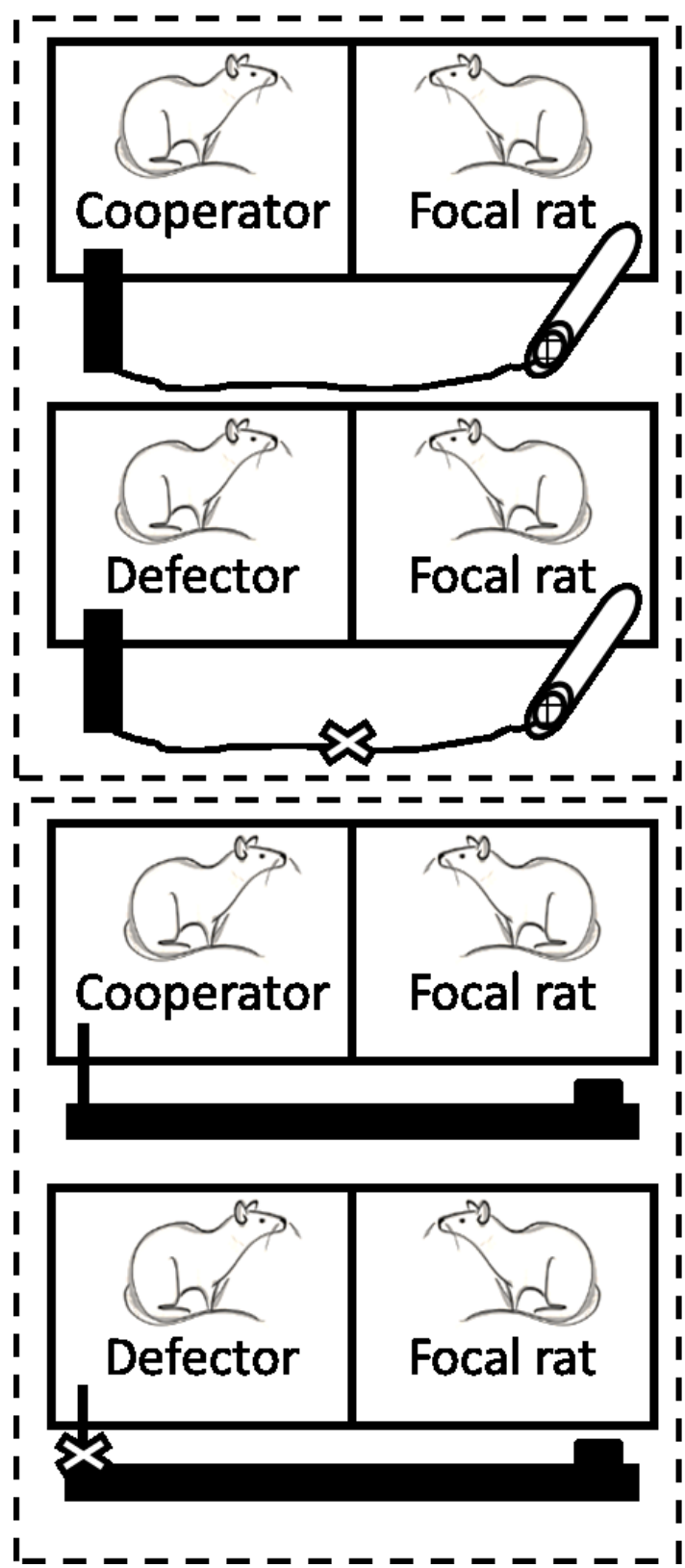

Test phase

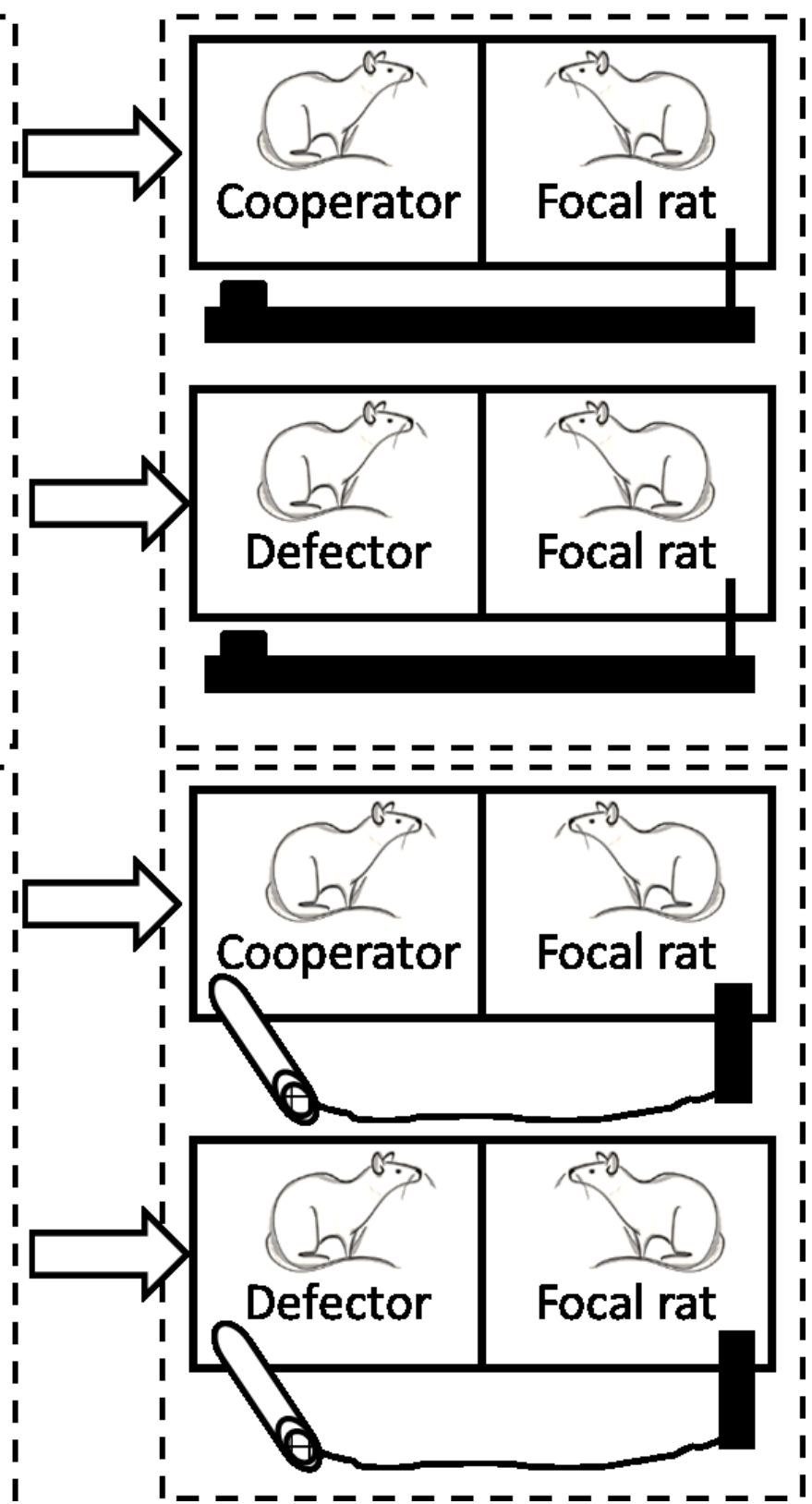

Figure 1. Test procedure

In the experience phase, focal rats either experienced a cooperator producing rewards for them by pulling a platform into the focal rat's compartment or by pushing a lever, or they experienced a defector that did not produce food for them with either mechanism. In the test phase, the roles were exchanged and focal rats could produce food for their social partner by using the alternative mechanism. Each focal rat was tested in all four situations with four different partners. 


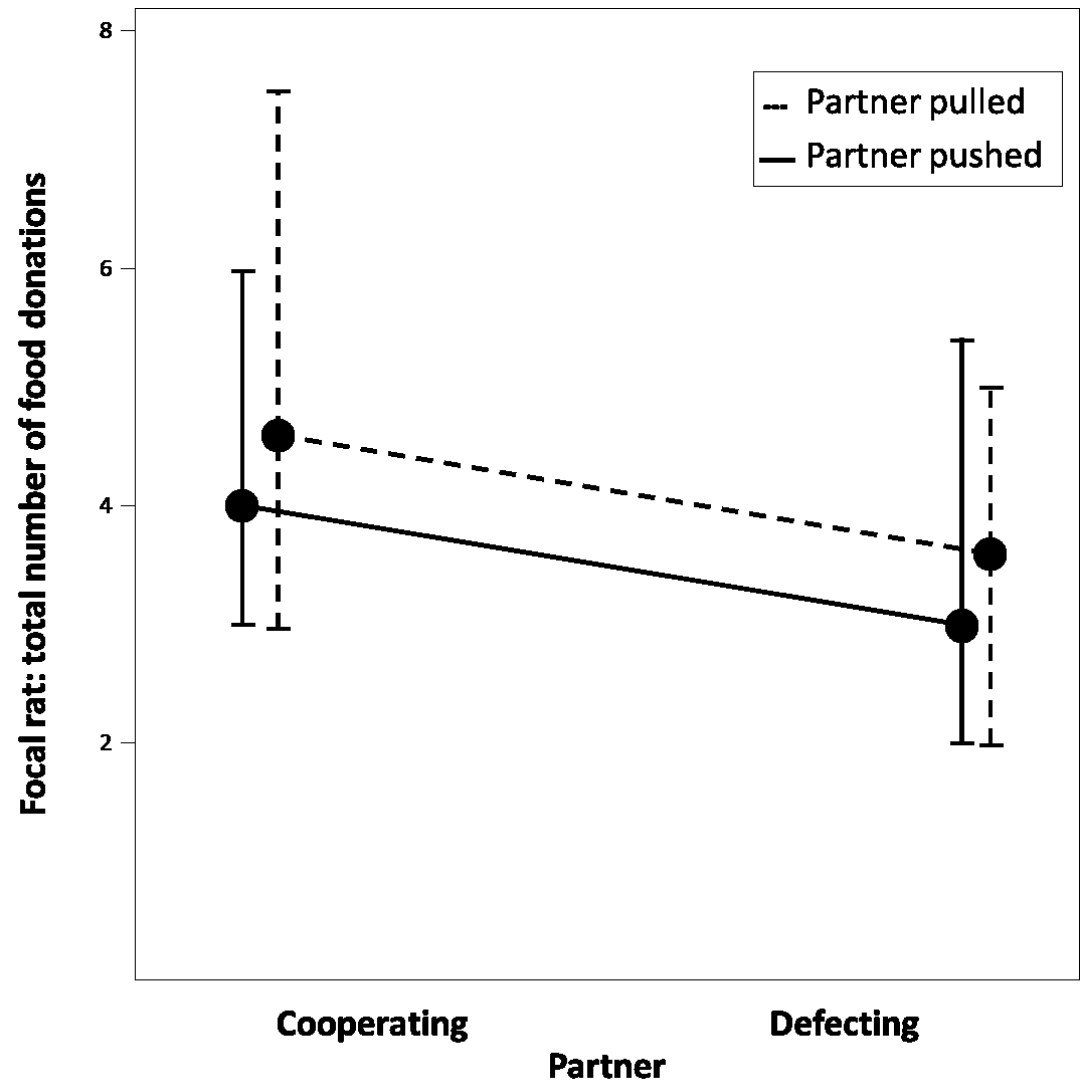

Figure 2. Help provided by focal rats to previously cooperating and defecting partners

Medians and interquartile ranges of food donated by focal rats during the test phase to partners that previously cooperated or defected by using the 'pulling' (dashed line) or 'pushing' (solid line) task are shown. 


\section{Appendix}

Latency to start donating food to the social partner

Besides the amount of help provided, the latency until the first food item is donated has been shown to indicate the motivation of rats to cooperate (Dolivo \& Taborsky, 2015; Schneeberger, Dietz, \& Taborsky, 2012). Consequently, we checked whether the latency to start providing food for cooperators was shorter than that for providing food to defectors. However, this was not the case, as found also in a previous study testing for generalized reciprocity (Rutte \& Taborsky, 2007). The timing of food provisioning in our experiment might not easily indicate the motivation of focal rats to cooperate. Focal rats pulled earlier than they pushed, suggesting that rats preferred to help by this device than by the other. This may be explained by the different force rats must exert to use each task. To push the lever rats had to exert the force of $0.24 \mathrm{~N}$, whereas to pull the stick they just needed $0.14 \mathrm{~N}$. Further, in contrast to previous studies, here focal rats were provided with a different device to their partners; therefore, the latency to the first food donation may not represent well the motivation to help the partner. However, although the latencies to start helping defectors and cooperators did not differ, the quantity of help provided clearly diverged (see Fig. 2). 


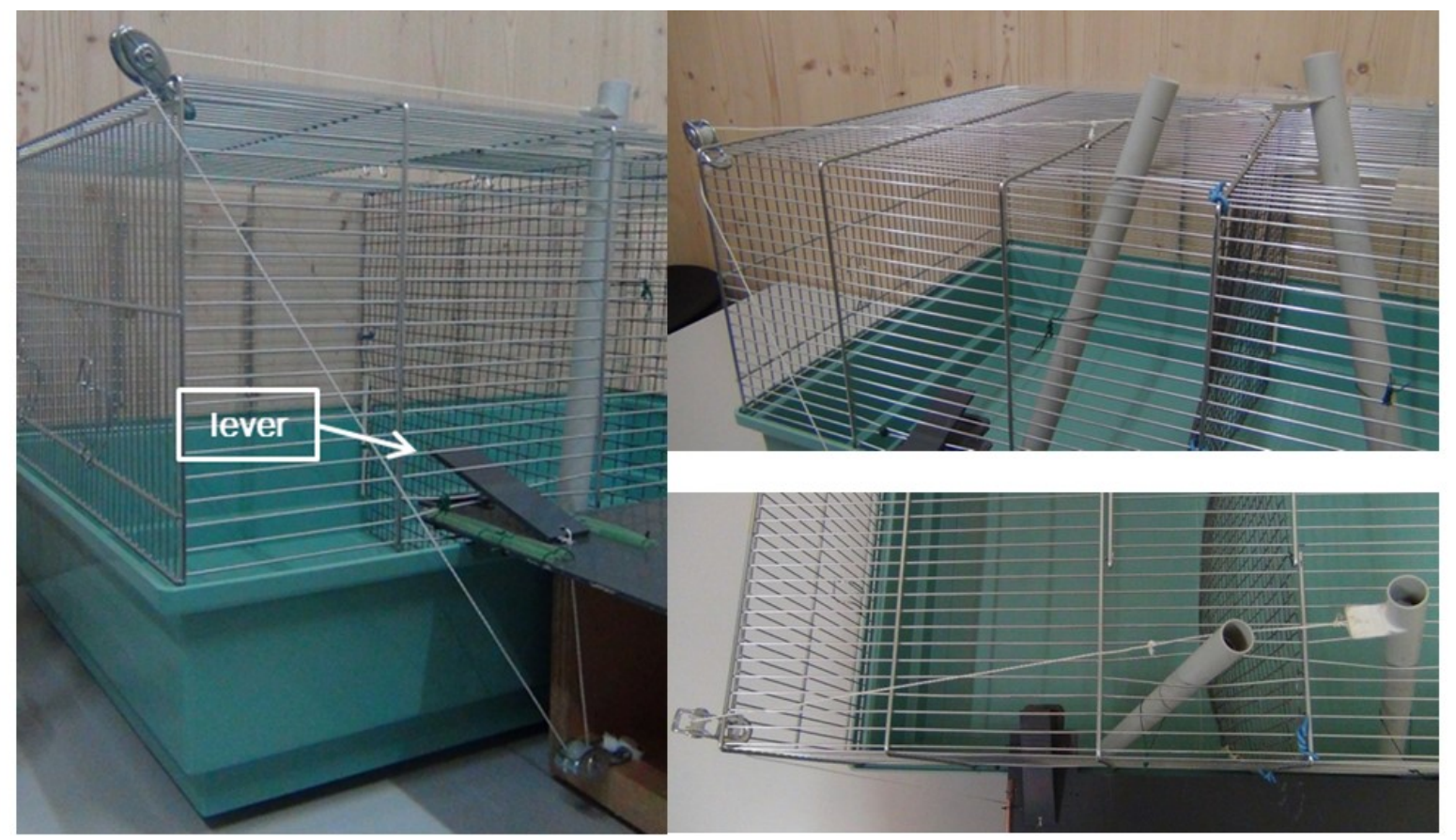

Figure A1. Pushing mechanism

When the rat pushed the lever, a trapdoor in the grey plastic tube opened releasing a food item, which fell into the partner's compartment. 


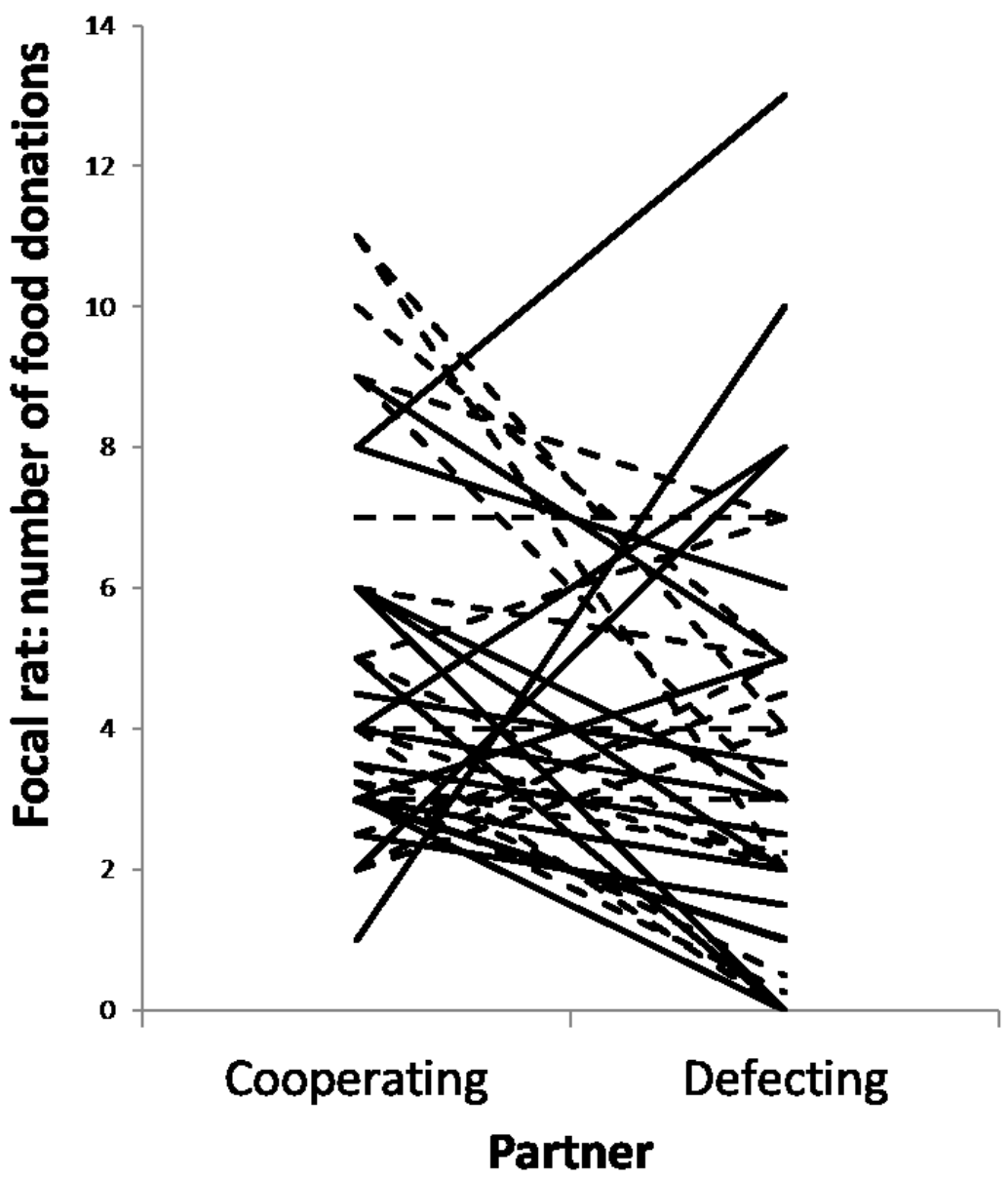

Figure A2. Help provided by each individual focal rat to previously cooperating and defecting partners

The graph shows the frequency of donations provided by focal individuals to partners that had used either the pulling (indicated by solid lines) or the pushing mechanism (indicated by dashed lines) during the experience phase. Because some lines completely overlapped, we raised eight of them slightly to make them visible. 


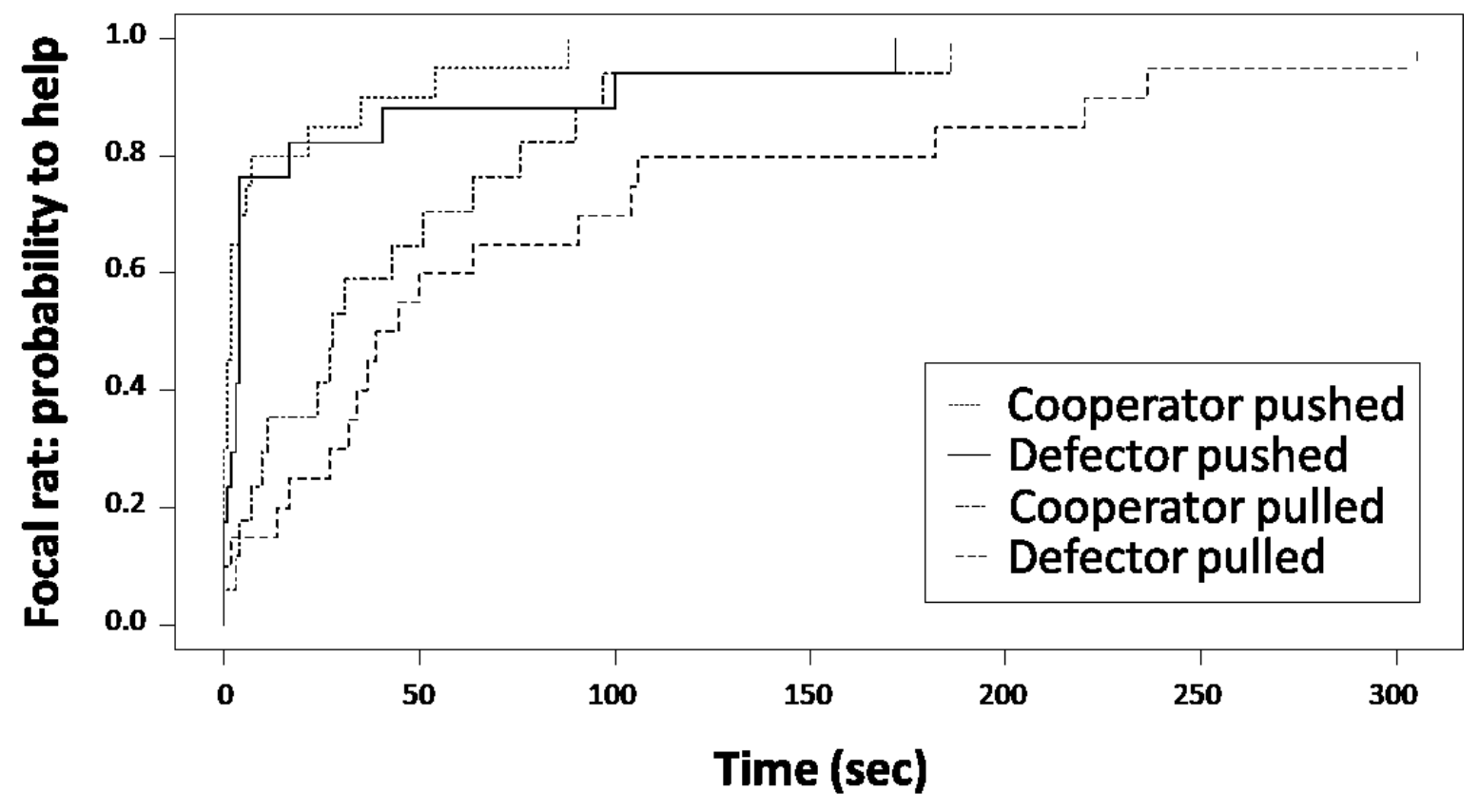

Figure A3. Latency to the first donation given by focal rats to previously helpful partners

The Kaplan-Meier plots show the calculated cumulative probability to help in relation to the time course of the experiment. 